\title{
Genetic diversity and population structure in Harpadon nehereus based on sequence- related amplified polymorphism markers
}

\author{
Z.H. Zhu*, H.Y. Li*, Y. Qin and R.X. Wang \\ Laboratory for Marine Living Resources and Molecular Engineering, \\ College of Marine Science, Zhejiang Ocean University, Zhoushan, China \\ *These authors contributed equally to this study. \\ Corresponding author: R.X. Wang \\ E-mail: wangrixin1123@126.com
}

Genet. Mol. Res. 13 (3): 5974-5981 (2014)

Received June 18, 2013

Accepted December 13, 2013

Published August 7, 2014

DOI http://dx.doi.org/10.4238/2014.August.7.13

\begin{abstract}
In this study, the genetic diversity among ten populations of the Bombay duck was studied on the basis of sequence-related amplified polymorphism (SRAP). The ten populations were collected from the East China Sea and South China Sea areas. A total of 98 loci were obtained from 292 individuals using eight SRAP primers. The average proportion of polymorphic loci, genetic diversity $(H)$, and Shannon's information index were $75.20 \%, 0.2478$, and 0.3735 , respectively. Nei's genetic distance and Shannon's information index between the ten populations ranged from 0.0410 to 0.3841 and from 0.2396 to 0.4506 , and the averages Nei's gene diversity index $(H=$ $0.2478)$ and Shannon's information index $(I=0.3735)$ at the population level were high. AMOVA showed that most of the variation was within populations $(71.74 \%)$, and only $28.26 \%$ of the variation was between populations. The neighbor-joining tree based on genetic distance revealed that significant genealogical structure existed throughout the examined range of the Bombay duck. The results demonstrated that SRAP marker was an effective tool for the assessment of genetic
\end{abstract}


diversity in the Bombay duck. The results could be used for further protection of the germplasm resource of the Bombay duck.

Key words: Harpadon nehereus; Genetic diversity; SRAP

\section{INTRODUCTION}

Harpadon nehereus, which is known as the Bombay duck, belongs to the Synodontidae. This species is widely distributed along the coast of China. Generally, H. nehereus attains a length of 15-25 cm and a weight of 75-100 g. The main fishing seasons of H. nehereus are spring and autumn. This species inhabits shallow, muddy and undersea environment. It is a kind of carnivorous fish that eats small fishes and benthic animals. At the same time, it is an important commercial fish for domestic use because of its delicious taste and value-added meat quality. Han et al. (1997) had done the development study on the seasoning of $H$. nehereus. A biorthogonal design was used to obtain the best quality indices of the analysis about $H$. nehereus.

$H$. nehereus is a highly valued marine fish species, and the resources of $H$. nehereus are also at a high level. For example, Lin (2009) studied the spatial distribution and environmental characteristics of $H$. nehereus in the East China Sea area and drew the conclusion that the estimated biomass was 2125 tons, but that potential biomass could surpass 5000 tons or more. However, the trophic level of this species has declined since the 1980s. It has dropped from 4.5 in the 1980 s to 3.8 at present. The germplasm resources of $H$. nehereus have been reduced maybe because of overfishing and overexploitation (Pan and Cheng, 2011).

In recent years, a number of studies on $H$. nehereus have been carried out, but most of these are related to microbial and biochemical composition, the number of aquatic products processed, spatial distribution, and feeding habits (Bhat and Albuquerque, 1953; Chen, 2000; Chen et al., 2004; Tumuluru et al., 2008; Lin, 2009). Nevertheless, despite the importance of $H$. nehereus as a commercial fish, not much information on its population genetics using molecular markers has been reported. To investigate the genetic characteristics of $H$. nehereus, we developed and characterized microsatellite markers, but only five polymorphic microsatellite loci were detected from 28 microsatellite loci. The low degree of polymorphism of microsatellite marker limits the investigation and analysis of genetic diversity in H. nehereus (Xu et al., 2011). Among various molecular markers, sequence-related amplified polymorphism (SRAP) markers are one of the most commonly used molecular markers, which have been successfully used in revealing population genetic diversity because they have several intrinsic characteristics, such as simplicity, reliability and good distribution in the genome (Li et al., 2010).

SRAP is a newly developed method that is based on two-primer amplifications, preferentially amplifying open reading frames ( $\mathrm{Li}$ and Quiros, 2001). The first type of primer (forward) is $17 \mathrm{bp}$ long containing a fixed sequence of 14 nucleotides rich in $\mathrm{C}$ and $\mathrm{G}$. It has three selective bases at the 3 '-end. This primer preferentially amplifies exonic regions, which tend to be CG rich. The second type of primer (reverse) is $18 \mathrm{bp}$ long, which contains a sequence of 15 nucleotides and is rich in A and T. It has three selective bases at the 3 '-end. This primer preferentially amplifies intronic regions and regions with promoters, which tend to be AT rich. The observed polymorphism fundamentally originates in the variation of the length of these introns, promoters and spacers both between individuals and between species (Li and Quiros, 2001; Ferriol et al., 2003; Wang et al., 2005; Dong et al., 2010). Compared with other molecu- 
lar markers, SRAP markers are more reproducible and stable. They are not complicated and can be used in different materials according to their unique primer design. Nowadays, SRAPs have been adapted for a variety of research purposes, including germplasm identification, linkage map construction, gene tagging, mapping, map-based cloning, and evolutionary study (Li and Quiros, 2001; Ferriol et al., 2003; Budak et al., 2004; Han et al., 2008; Mutlu et al., 2008; Lin et al., 2004, 2009). The objective of our study was to assess the genetic diversity within populations and the differentiation between populations of $H$. nehereus. This information will in turn provide an important theoretical basis for the protection and sustainable utilization of other important edible species.

\section{MATERIAL AND METHODS}

\section{Sample collection and genomic DNA extraction}

A total of 292 samples of $H$. nehereus were collected from ten geographic locations belonging to two sea areas, the seven locations of Zhoushan (ZS), Xiangshan (XS), Ninghai $(\mathrm{NH})$, Yueqing (YQ), Wenling (WL), Wenzhou (WZ), Ruian (RA) are geared to the East China Sea area; the other populations from Xiamen (XM), Zhuhai (ZH) and Haikou (HK) belong to the South China Sea area (Figure 1). Thirty individuals were selected per population except $\mathrm{HK}$, which only had 22 individuals. These tissue samples were obtained from fin clips and preserved in $95 \%$ ethanol, and finally stored at $-20^{\circ} \mathrm{C}$. Total genomic DNA was isolated from the caudal fins using the standard phenol-chloroform method, with some modification. The results were checked by $1 \%$ agarose gel electrophoresis. Afterwards, the DNA samples were subsequently dissolved in $100 \mu \mathrm{L}$ TE buffer and kept at $4^{\circ} \mathrm{C}$ for later use.

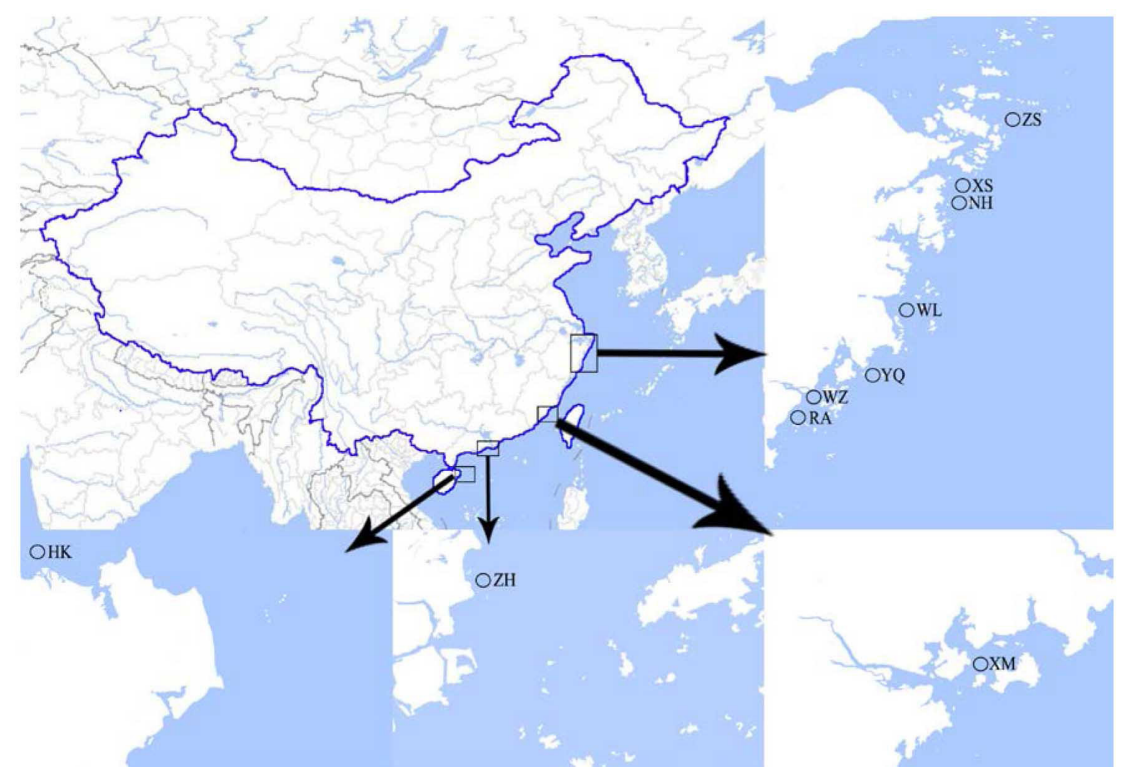

Figure 1. Sample sites for Harpadon nehereus. $\mathrm{HK}=$ Haikou; $\mathrm{ZH}=\mathrm{Zhuhai} ; \mathrm{XM}=\mathrm{Xiamen}$; $\mathrm{RA}=$ Ruian; $\mathrm{WZ}=$ Wenzhou; $\mathrm{YQ}=$ Yueqing; $\mathrm{WL}=$ Wenling; $\mathrm{NH}=$ Ninghai; $\mathrm{XS}=$ Xiangshan; $\mathrm{ZS}=$ Zhoushan. 


\section{PCR amplification and SRAP analysis}

From 64 primer combinations, eight combinations of primer pairs, which were synthesized according to the description by Li and Quiros (2001), were chosen to be used for SRAP analysis (Table 1). The PCR amplifications were carried out in a $15-\mu \mathrm{L}$ volume consisting of $10 \mu \mathrm{L}$ sterilized water, $1.5 \mu \mathrm{L} \mathrm{10X} \mathrm{PCR} \mathrm{buffer} \mathrm{(containing} 1.5 \mathrm{mM} \mathrm{Mg}^{2+}$ ), $1.2 \mu \mathrm{L} 2.5 \mathrm{mM}$ dNTPs, $0.6 \mu \mathrm{L}$ forward or reverse primer, $1 \mu \mathrm{L}$ diluted DNA template, and $0.1 \mu \mathrm{L} 5 \mathrm{U}$ Taq DNA polymerase (Tiangen, Beijing, China). PCR was performed using the following schedule: initial denaturation at $94^{\circ} \mathrm{C}$ for $5 \mathrm{~min}$, followed by 5 cycles of denaturation at $94^{\circ} \mathrm{C}$ for $1 \mathrm{~min}, 35^{\circ} \mathrm{C}$ for $1 \mathrm{~min}$ and $72^{\circ} \mathrm{C}$ for $1 \mathrm{~min}, 35$ cycles of denaturation at $94^{\circ} \mathrm{C}$ for $1 \mathrm{~min}, 53^{\circ} \mathrm{C}$ for $1 \mathrm{~min}$, and extension at $72^{\circ} \mathrm{C}$ for $1 \mathrm{~min}$, and a final extension at $72^{\circ} \mathrm{C}$ for $10 \mathrm{~min}$, finally holding at $4^{\circ} \mathrm{C}$. PCR amplification was performed with an ABI 9700 and DNA fragments ranging from 100 to $2000 \mathrm{bp}$ were separated on a $1.5 \%$ agarose gel.

\section{Table 1. Sequences of forward and reverse SRAP primers of PCR amplification.}

\begin{tabular}{lll}
\hline Primer & Type & Sequence $\left(5^{\prime}-3^{\prime}\right)$ \\
\hline Me 1 & Forward & TGAGTCCAAACCGGATA \\
Me 2 & Forward & TGAGTCCAAACCGGAGC \\
Me 3 & Forward & TGAGTCCAAACCGGAAT \\
Me 5 & Forward & TGAGTCCAAACCGGAAG \\
Em 1 & Reverse & GACTGCGTACGAATTAAT \\
Em 2 & Reverse & GACTGCGTACGAATTTGC \\
Em 3 & GACTGCGTACGAATTGAC \\
Em 4 & Reverse & GACTGCGTACGAATTTGA \\
Em 7 & Reverse & GACTGCGTACGAATTCAA \\
\hline
\end{tabular}

\section{Data analyses}

In the SRAP molecular analysis, the amplified fragments were scored as either present (1) or absent (0) for each primer pair, where (1) stands for the presence and (0) stands for the absence for each SRAP fragment. Only reproducible and clear bands were selected. The data were transformed into 0/1 binary character matrix (Yang et al., 2010). The number of polymorphic loci $(P O L)$, the percentage of polymorphic loci $(P P B)$ and heterozygosity $(H)$ for every population were estimated using POPGENE32 (Yeh and Boyle, 1997). Genetic distance and Shannon's index $(I)$ were also calculated to characterize the gene diversity and the distribution of the variation using the same program. Based on Nei's standard genetic distance (Nei, 1972), a neighbor-joining (NJ) tree was constructed using the FigTree software. Analysis of molecular variance (AMOVA) was carried out using the Arlequin 3.11 software to estimate how variation was partitioned within and between populations (Excoffier, 1993).

\section{RESULTS AND DISCUSSION}

Genetic diversity parameters for every population are presented in Table 2. A total of eight SRAP primer combinations were screened and a total of 98 loci were obtained in 
the ten populations studied. The POLs were spread from 52 to 83 , with an average of 73.7. The $P P B s$ for populations varied from $53.06 \%$ in the HK population to $84.69 \%$ in the YQ population, with an average of $75.20 \%$. A high percentage of polymorphism observed with these markers indicated the existence of a high level of genetic diversity between different populations of $H$. nehereus. This indication showed that the genetic exchanges between these populations were relatively frequent. This could be calculated maybe because of the great number of $H$. nehereus in the coastal areas of China and the geographic isolation. The average value of $I$ was 0.3735 at the population level. This result also showed the high level of genetic diversity existing in the populations of $H$. nehereus studied.

\begin{tabular}{|c|c|c|c|c|}
\hline Population & $P O L$ & $P P B(\%)$ & $H$ & $I$ \\
\hline XM & 79 & 80.61 & 0.2740 & 0.4111 \\
\hline $\mathrm{ZH}$ & 79 & 80.61 & 0.2934 & 0.4345 \\
\hline XS & 71 & 72.45 & 0.2126 & 0.3259 \\
\hline YQ & 83 & 84.69 & 0.3026 & 0.4506 \\
\hline $\mathrm{NH}$ & 81 & 82.65 & 0.2978 & 0.4426 \\
\hline HK & 52 & 53.06 & 0.1657 & 0.2525 \\
\hline WL & 82 & 83.67 & 0.2654 & 0.4017 \\
\hline RA & 78 & 79.59 & 0.2725 & 0.4077 \\
\hline $\mathrm{ZS}$ & 55 & 56.12 & 0.1540 & 0.2396 \\
\hline WZ & 77 & 78.57 & 0.2398 & 0.3690 \\
\hline Average & 73.7 & 75.20 & 0.2478 & 0.3735 \\
\hline
\end{tabular}

$P O L=$ number of polymorphic loci; $P P B=$ percentage of polymorphic loci; $H=$ gene diversity (Nei, 1972); $I=$ Shannon's information index. For population abbreviations, see legend to Figure 1.

Although the results above showed that the genetic diversity of $H$. nehereus is relatively rich, the number of polymorphic loci in the HK and ZS populations was only 52 and 55, respectively, which were substantially lower than in the other 8 populations. The percentage of polymorphic loci in the HK population was the lowest among the ten populations studied, followed by the ZS population. The other populations with higher percentages were nearly at the same level. So we should pay more attention to the HK and ZS populations for the further protection of germplasm resources because of their counter - low genetic diversity level. From all the data above, this difference could be the result of the difference in genetic composition of the populations studied, or the great selective power of SRAP polymorphism. By using SRAP markers, this study also showed that there is a high level of genetic diversity between populations and low level of genetic variations within populations of $H$. nehereus.

Nei's gene diversity $(H)$ of the ten populations (XM, ZH, XS, YQ, NH, HK, WL, RA, ZS, and WZ) was 0.2740, 0.2934, 0.2126, 0.3026, 0.2978, 0.1657, 0.2654, 0.2725, 0.1540 , and 0.2398 , respectively (Table 2 ). It was suggested that the NH population had the highest level of genetic diversity, followed by the YQ population. Shannon's information index $(I)$ of the ten populations was $0.4111,0.4345,0.3259,0.4506, .4426,0.2525$, $0.4017,0.4077,0.2396$, and 0.3690 , respectively. The results revealed that the genetic diversity of the NH and YQ population is rich. At the same time, from the data above, we found that the $H$ values in the HK and ZS populations were lower than in the other eight populations, but the overall values showed that genetic diversity was relatively high be- 
tween all these ten populations. This finding is the same as in the analysis of $P O L$ and $P P B$. Pairwise distances calculated with the index revealed that there are clear differences between populations (Table 3). The genetic distance between the ZS and HK populations was the highest, up to 0.3841 , while that between the ZS and WZ populations was the lowest at 0.0410 . The result demonstrated that the farthest relationship existed between the ZS and HK populations and the lowest genetic distance existed between the ZS and WZ populations. The genetic distance corresponded exactly to the geographical distribution of $H$. nehereus.

Table 3. Genetic identity and genetic distance of ten populations of Harpadon nehereus.

\begin{tabular}{|c|c|c|c|c|c|c|c|c|c|c|}
\hline Population & $\mathrm{XM}$ & $\mathrm{ZH}$ & XS & YQ & $\mathrm{NH}$ & HK & WL & RA & $\mathrm{ZS}$ & WZ \\
\hline XM & $* * * *$ & 0.9349 & 0.8640 & 0.8795 & 0.8815 & 0.8794 & 0.8876 & 0.9079 & 0.7586 & 0.8395 \\
\hline $\mathrm{ZH}$ & 0.0673 & $* * * *$ & 0.8627 & 0.8856 & 0.8792 & 0.8665 & 0.8795 & 0.9099 & 0.7362 & 0.8486 \\
\hline XS & 0.1462 & 0.1477 & $* * * *$ & 0.9319 & 0.9019 & 0.7844 & 0.9472 & 0.9598 & 0.8903 & 0.9501 \\
\hline YQ & 0.1284 & 0.1215 & 0.0706 & $* * * *$ & 0.9219 & 0.8331 & 0.9493 & 0.9415 & 0.8741 & 0.9400 \\
\hline $\mathrm{NH}$ & 0.1261 & 0.1287 & 0.1033 & 0.0813 & $* * * *$ & 0.8376 & 0.9024 & 0.9019 & 0.8191 & 0.8943 \\
\hline HK & 0.1285 & 0.1433 & 0.2429 & 0.1826 & 0.1772 & $* * * *$ & 0.8145 & 0.8319 & 0.6810 & 0.7848 \\
\hline WL & 0.1192 & 0.1285 & 0.0543 & 0.0521 & 0.1027 & 0.2052 & $* * * *$ & 0.9498 & 0.8832 & 0.9544 \\
\hline RA & 0.0966 & 0.0945 & 0.0410 & 0.0603 & 0.1033 & 0.1840 & 0.0515 & $* * * *$ & 0.8634 & 0.9525 \\
\hline ZS & 0.2763 & 0.3062 & 0.1162 & 0.1346 & 0.1995 & 0.3841 & 0.1243 & 0.1469 & $* * * *$ & 0.9059 \\
\hline WZ & 0.1750 & 0.1641 & 0.0511 & 0.0618 & 0.1117 & 0.2423 & 0.0467 & 0.0486 & 0.0988 & $* * * *$ \\
\hline
\end{tabular}

Nei’s genetic identity (above diagonal) and genetic distance (below diagonal).

The genetic variation based on AMOVA showed that $71.74 \%$ of genetic variation resided within populations, while it was only $28.26 \%$ between populations (Table 4 ). This result suggested the presence of a significant geographical division and indicated a stable regional genetic structure in this species. Thus, we could state that there is an evident variation within populations.

Table 4. Analysis of molecular variance (AMOVA) for populations of Harpadon nehereus.

\begin{tabular}{lrcccc}
\hline Source of variation & d.f. & Sum of squares & Variance components & Percentage of variation & $F_{\text {ST }}$ \\
\hline Between populations & 9 & 1095.967 & 4.5864 & 28.26 \\
Within populations & 231 & 2689.024 & 11.6408 & 71.74 \\
Total & 240 & 3784.992 & 16.2272 & & 0.2826 \\
\hline
\end{tabular}

d.f. $=$ degrees of freedom.

On the basis of the interpopulation genetic distance, the NJ tree was constructed, which is shown in Figure 2. According to the genetic distance, the NJ cluster analysis indicated the existence of two main clusters. The first cluster was composed of seven populations from ZS, WZ, XS, WL, RA, YQ, and NH, which belong to the East China Sea area; the second cluster is composed of three populations from $\mathrm{ZH}, \mathrm{XM}$ and HK, which are in the South China Sea. This sorting corresponded to the geographic division. That is to say, the samples not only could be distinguished from each other between different populations, but also could be easily separated between the two sea areas.

In conclusion, all ten populations of $H$. nehereus were found to have high genetic diversity and all indices studied could provide a useful theoretical basis. Also, these results suggest that the SRAP marker method can be used in the protection of germplasm resources for this species. 


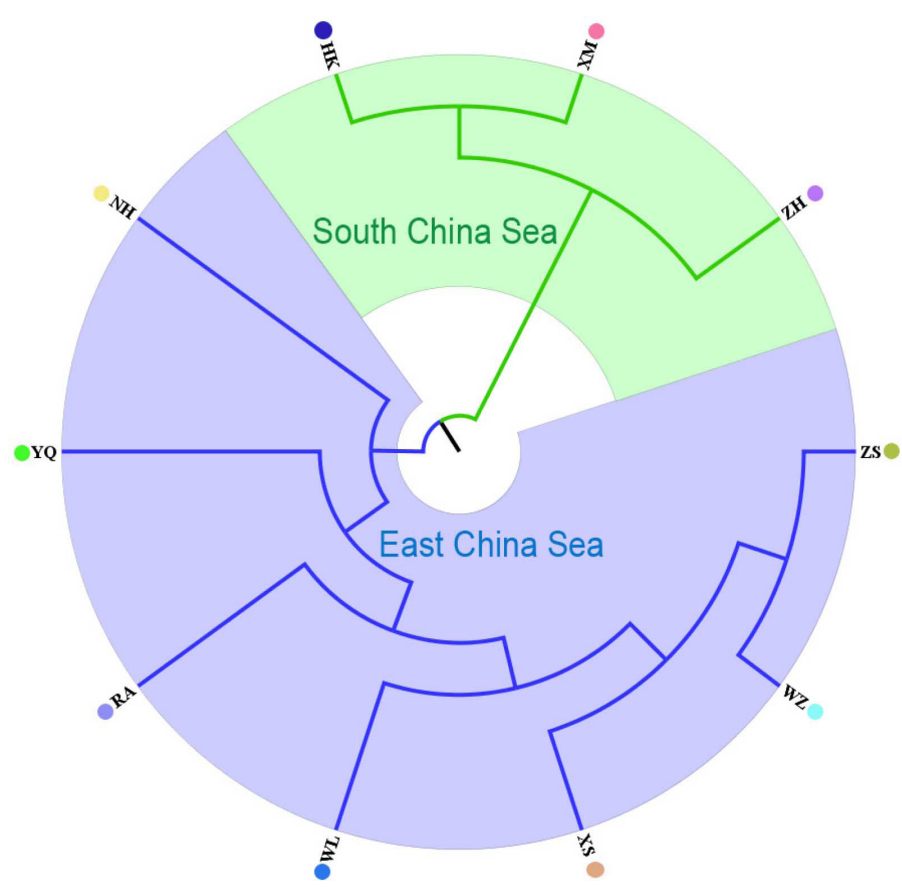

Figure 2. Molecular phylogenetic tree of ten populations of Harpadon nehereus. For population abbreviations, see legend to Figure 1.

\section{ACKNOWLEDGMENTS}

Research supported by the Zhejiang Province Science and Technology Project (\#2010R50025).

\section{REFERENCES}

Bhat JV and Albuquerque MJ (1953). Micro-organisms associated with the "Bombay Duck" or Bombil (Harpodon nehereus Buch). Plant Sci. 38: 101-108.

Budak H, Shearman RC, Parmaksiz I, Gaussoin RE, et al. (2004). Molecular characterization of buffalo grass germplasm using sequence-related amplified polymorphism markers. Theor. Appl. Genet. 108: 328-334.

Chen HQ, Jiao HF, Zhu JQ and Ouyang ZY (2004). Configurational character and digestive system of Harpodon nehereus. J. Ningbo Univ. 17: 389-392.

Chen XX (2000). Processing technology of Harpodon nebereus fillet. Mar. Sci. 24: 10-12.

Dong P, Wei YM, Chen GY, Li W, et al (2010). Sequence-related amplified polymorphism (SRAP) of wild emmer wheat (Triticum dicoccoides) in Israel and its ecological association. Biochem. Syst. Ecol. 38: 1-11.

Excoffier L (1993). Analysis of Molecular Variance (AMOVA). Version 1.55. Genetics and Biometry Laboratory, University of Geneva, Geneva.

Ferriol M, Pico B and Nuez F (2003). Genetic diversity of a germplasm collection of Cucurbita pepo using SRAP and AFLP markers. Theor. Appl. Genet. 107: 271-282.

Han SZ, Tang DJ and He YB (1997). Development study of on the seasoning of Harpodon nehereus. J. Zhe Col. Fish. 16: $195-200$

Han XY, Wang LS, Liu ZA, Jan DR, et al (2008). Characterization of sequence-related amplified polymorphism markers 
analysis of tree peony bud sports. Sci. Hort. 115: 261-267.

Li AX, Liu QC, Wang QM, Zhang LM, et al (2010). Construction of molecular linkage maps using SRAP markers in sweet potato. Acta. Agr. Sin. 36: 1286-1295.

Li G and Quiros CF (2001). Sequence-related amplified polymorphism (SRAP), a new marker system based on a simple PCR reaction: its application to mapping and gene tagging in Brassica. Theor. Appl. Genet. 103: 455-461.

Lin LS (2009). Spatial distribution and environmental characteristics of Harpodon nehereus in the East China Sea region. J. Shang Ocean Univ. 18: 66-71.

Lin ZX, Zhang XL and Nie YC (2004). Evaluation of application of a new molecular marker SRAP on analysis of F2 segregation population and genetic diversity in cotton. Acta Genet. Sin. 31: 622-626.

Lin ZX, Zhang YX, Zhang XL and Guo XP (2009). A high-density integrative linkage map for Gossypium hirsutum. Euphytica 166: 35-45.

Mutlu N, Boyacı FH, Göçmen M and Abak K (2008). Development of SRAP, SRAP-RGA, RAPD and SCAR markers linked with a Fusarium wilt resistance gene in eggplant. Theor. Appl. Genet. 117: 1303-1312.

Nei M (1972). Genetic distance between populations. Am. Nat. 106: 283-292.

Pan XW and Cheng J-H (2011). Feeding ecology of Harpadon nehereus in areas adjacent to Changjiang River estuary. $J$. Fish. Sci. Chin. 18: 1132-1140.

Tumuluru JS, Sokhansanj S, Sukumar B and Bawa AS (2008). Storage properties of low fat fish and rice flour coextrudates. Food Bioprocess Technol. 3: 481-490.

Wang G, Pan JS, Li XZ, He HL, et al. (2005). Construction of a cucumber genetic linkage map with SRAP markers and location of the genes for lateral branch traits. Sci. Chin. Ser. 48: 213-220.

Xu TJ, Sun DQ, Li HY and Wang RX (2011). Development and characterization of microsatellite markers for the lizardfish known as the Bombay duck, Harpadon nehereus (Synodontidae). Genet. Mol. Res. 10: 1701-1706.

Yang P, Chen LQ, Wang W, Yu N, et al. (2010). Genetic diversity of oriental river prawn (Macrobrachium nipponense De Haan) revealed by ISSR markers. J. Fish. Sci. Chin. 17: 913-921.

Yeh FC and Boyle TJB (1997). Population genetic analysis of co-dominant and dominant markers and quantitative traits. Bel. J. Bot. 129:157. 